\title{
Anaesthesia management for bronchoscopic and surgical lung volume reduction
}

\author{
Bastian Grande ${ }^{1}$, Torsten Loop ${ }^{2}$ \\ ${ }^{1}$ Institute of Anaesthesiology, University Hospital Zurich, Switzerland; ${ }^{2}$ Department of Anaesthesiology and Intensive Care Medicine, University \\ Medical Center, Freiburg, Germany \\ Contributions: (I) Conception and design: All authors; (II) Administrative support: None; (III) Provision of study materials or patients: None; (IV) \\ Collection and assembly of data: None; (V) Data analysis and interpretation: None; (VI) Manuscript writing: All authors; (VII) Final approval of \\ manuscript: All authors. \\ Correspondence to: Bastian Grande, MD. University Hospital Zurich, Raemistreet 100, 8097 Zurich, Switzerland. Email: bastian.grande@usz.ch.
}

\begin{abstract}
Optimizing the patient's condition before the lung volume reduction (LVR) according to recommendations by American College of Cardiology/American Heart Association (ACC/AHA) guideline on perioperative cardiovascular evaluation is mandatory. Implementation of a multimodal analgesia concept and the use short-acting anaesthetics enhances recovery and avoids postoperative pulmonary complications. Normovolemia, normothermia, lung protective ventilation and an evidence-based concept of airway management (i.e., double-lumen tube, bronchus blocker) are suggested for intraoperative management of surgical lung volume reduction (SLVR). General anaesthesia (using remifentanil, propofol and mivacurium) with an i-gel ${ }^{\circledR}$ supraglottic airway device should be used for bronchoscopic lung volume reduction (BLVR). Jet ventilation through rigid bronchoscopy or with a jet catheter may be an alternative concept. Experienced consultants should perform anaesthesia for LVR.
\end{abstract}

Keywords: Lung volume reduction surgery (LVRS); bronchoscopic lung volume reduction (BLVR); anaesthesia management

Submitted Dec 21, 2017. Accepted for publication Feb 09, 2018.

doi: $10.21037 /$ jtd.2018.02.46

View this article at: http://dx.doi.org/10.21037/jtd.2018.02.46

\section{Introduction}

Reducing hyperinflated diseased lung in patients suffering from inhomogeneous emphysema was introduced more than 50 years ago (1). Lung volume reduction (LVR) includes surgical methods, so-called lung volume reduction surgery (LVRS) and bronchoscopic lung volume reduction (BLVR).

Anaesthesia management for LVR procedures is challenging. Besides severe airway obstruction and impaired gas exchange, patients undergoing LVR often suffer from pre-existing comorbidity and their physical status of the American Society of Anaesthesiology classification is higher than three (2). The operation site and the mechanical ventilation are interdependent and lung protective mechanical ventilation of the previously diseased lung is crucial for the postoperative success of the procedure. Recovery of spontaneous breathing at the end of surgery and extubation are mandatory to reduce airway pressure (3). Implementation of a multimodal analgesia concept and the use short-acting anaesthetics enhanced recovery. In addition, optimal fluid therapy and active warming could achieve normovolemia and normothermia.

Anaesthesia for LVR is a challenge in these patients and should be performed by experienced consultants, since airway management for BLVR and LVRS is different and may be difficult: BLVR needs the sharing of the airway between interventionist and anaesthesiologist—LVRS needs one-lung-ventilation with lung separation. An algorithm for securing airway management should be established. Beyond the difference from established airway algorithms, training in difficult LVR airway management has been shown very 
effective (4).

\section{Preoperative management}

Optimizing the patient's condition before the LVR reduces postoperative complications and may be mandatory for successful treatment. First, assessment of individual patient risk factors before the planned intervention allows pretreatment optimization. Due to the risk factors and preexisting conditions of patients prior to the LVR, it is sometimes necessary to coordinate further interventions like coronary interventions prior to the LVR (5) recommended by American College of Cardiology/American Heart Association (ACC/AHA) guideline on perioperative cardiovascular evaluation and management of patients undergoing non-cardiac surgery (5). A recent transthoracic echocardiography should have been performed on each patient, especially to evaluate the right ventricular function. A systolic pulmonary arterial pressure (SPAP) $>35 \mathrm{mmHg}$ and with a homogeneous emphysema is considered as a contraindication for LVR (6). SPAP up to $45 \mathrm{mmHg}$ in patients with heterogeneous emphysema and severe hyperinflation could be tolerable for LVR, although robust evidence is missing (6).

Alcohol and smoking should be reduced and ceased preoperatively $(7,8)$ to reduce the risk of infections, respiratory, and neurological complications and the intensive care unit (ICU) stay (9). Whether preoperative spirometry training is beneficial in patients undergoing thoracotomy and lung resection (10) and despite lacking data it should be recommended in patients for LVR. As preoperative medication for anxiolysis, benzodiazepines seem to have advantages over alpha-2-agonists (11). However, anxiolysis must be carefully weighed towards sedative and respiratory depressive effects especially in the LVR patient collective.

\section{Anaesthesia management in LVRS}

\section{Strategy}

The main strategy in anaesthesia management for LVR should be an effective analgesic concept with combined methods, lung protective ventilation and an evidence based concept of airway separation. Better pain control with thoracic epidural anesthesia (TEA) is established in a wide range of surgical procedures (12). A recent meta-analysis of the pulmonary effects of TEA showed a reduced rate of pneumonia after TEA, probably due to earlier mobilization, reduced opioid consumption, and improved cough (13).

In the past, general anaesthesia and separated ventilation of the lungs were always necessary in thoracic surgery (1). The only way to perform lung-separation with one-lung ventilation has been a double-lumen tube or bronchus blockade. The bronchus blockade seemed to have disadvantages due to the over-inflated lungs of the patients undergoing LVR and has been rarely used (14). With the introduction of video-assisted thoracoscopic surgery other strategies such as non-intubated video-assisted thoracic surgery (NIVATS) may be an alternative concept, which has to been further proved to produce robust evidence by future well-designed studies (15). Without intubation, the spontaneously breathing patient develops a pneumothorax after surgical opening of the pleura, which may create sufficient operating conditions. However, the cough reflex must be prevented by surgical vagus blockade (16). The disadvantages are movements, conversion to open procedure, insufficient analgesia, panic attacks and respiratory complications $(15,16)$. The conversion rate is described as less than $5 \%(15)$.

\section{Anaesthetics}

The selection of short-acting anaesthetics in combination with thoracic epidural analgesia is important to reduce postoperative complications. On one hand, postoperative complications such as nausea and vomiting can be reduced, on the other hand, the avoidance of anaesthesia overhang is essential (17). Remifentanil as a short-acting opioid with a very short context-sensitive half-life seems to be a valid choice for anaesthesia maintenance, but no data on opioid choice in LVR patient exist. Due to the short half-life time of remifentanil and therefore a lack of postoperative effect, it should only be used as part of a multimodal pain concept.

The necessity of muscle relaxation, especially for the suppression of intraoperative coughing, should be performed with reversible muscle relaxants. Clear data on the save use of cisatracurium exists, but after the introduction of a selective binding agent for reversal of neuromuscular blockade-sugammadex-rocuronium bromide may be an alternative. Since postoperative recurarization is a morbidity factor in anaesthesia, antagonization is mandatory. Rocuronium shows slight advantages through the introduction of sugammadex, especially when neostigmine related side effects count. There were no significant differences in a recent single 
Table 1 Parameters of lung protective ventilation in LVRS

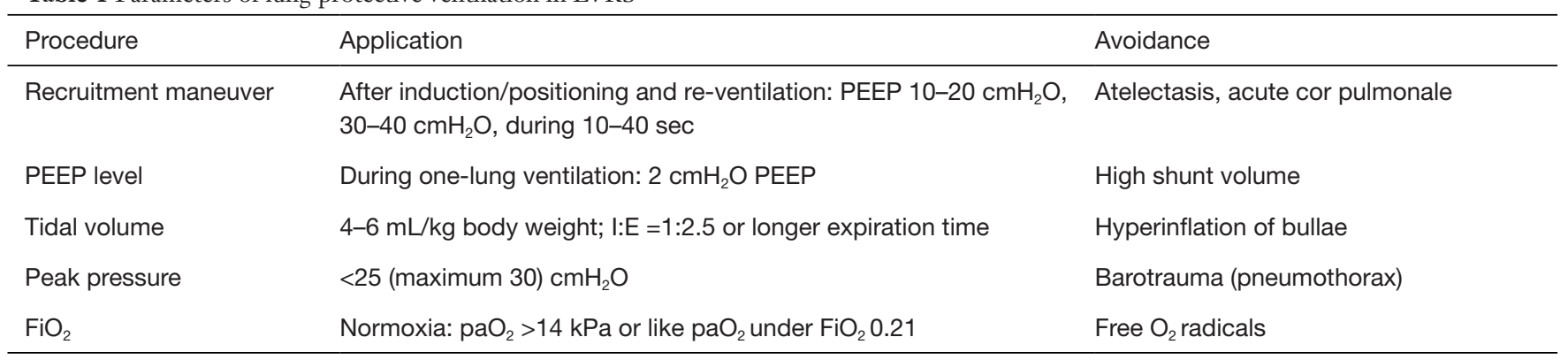

I:E means inspiratory time : expiratory time. LVRS, lung volume reduction surgery; PEEP, positive end-expiratory pressure.

blind trial of 120 patients if both are antagonized with neostigmine (18). The use of neuromuscular monitoring for muscle relaxation is strictly recommended, to especially avoid respiratory complications in LVR patients.

The use of intravenous or inhaled anaesthesia has long been the subject of numerous studies. In a randomized controlled multicentre trial of Beck-Schimmer et al. with 460 patients addressing the effect of volatile versus intravenous anaesthetics on major complications after lung surgery no difference between the two anaesthesia regimens was evident $(19,20)$. Importantly inhalational anaesthetics dose-dependently influence local inflammatory processes, partially reverse hypoxic pulmonary vasoconstriction and reduce alveolar clearance $(19,21)$. Due to the short half-life time and faster recovery anaesthesia with desflurane seems to be a preferable option.

\section{Lung protective ventilation}

Lung injury is the leading cause of death after thoracic surgery. Protective ventilation may prevent postoperative lung injury and reduces postoperative pulmonary complications. Elements of lung protective ventilation are short duration of ventilation (i.e., one-lung ventilation), low $\mathrm{FiO}_{2}$, low tidal volume [4-5 mL/kg body weight (BW)], recruitment after induction and after one-lung ventilation and moderate positive end-expiratory pressure (PEEP) levels $\left(5-10 \mathrm{cmH}_{2} \mathrm{O}\right.$ ) (see Table 1) (22).

Furthermore, permissive hypercapnia may be used to accelerate oxygen delivery during one-lung ventilation and in chronic obstructive pulmonary disease (COPD) patients with residual hyperinflation (3).

Lung protective ventilation has become an integral part of protocols to prevent pulmonary complications like pneumonia or accelerate the recovery time [e.g., enhanced recovery after surgery (ERAS)], which is both also, wanted in LVR (23-25).

One-lung-ventilation allows both open and video-assisted thoracic surgery. However, it also represents an independent risk factor for complications such as pneumonia, atelectasis and acute lung damage.

The two key steps during LVRS are re-expansion and ventilation of the suspended lung and early extubation. Reexpansion and ventilation of the operated lung should be carried out during lung separation because of the different compliance of both lungs. The concept 'open the lung and keep it open' could be a guide in re-ventilation although clear data for patients undergoing LVRS is missing (26). The extubation of patients with a double-lumen tube often leads to coughing, which may affect the outcome of the operation and lead to relevant pneumopleural fistula. For this reason, extubation of the patient in deep anaesthesia and relaxation, intubation with laryngeal mask (LM) second generation, transfer to spontaneous respiration and removal of the LM from the awake and spontaneously breathing patients should be recommended.

\section{Perioperative fluid management}

The amount and quality of perioperative fluids applicated treads the tight line between hypovolemic complications, especially acute kidney injury and fluid overload of the lungs (27). The situation increases by the scarce pulmonary functional reserves of the LVR patients. Strong evidence exists for a normovolemic fluid regimen in thoracic surgery (Table 2). Balanced crystalloids are recommended, saline solutions should be avoided and colloids should only be used as a substitute for blood loss $(23,25)$.

The integration of volume management into a "Patient Blood Management" tool shows many advantages 
Table 2 Perioperative fluid management

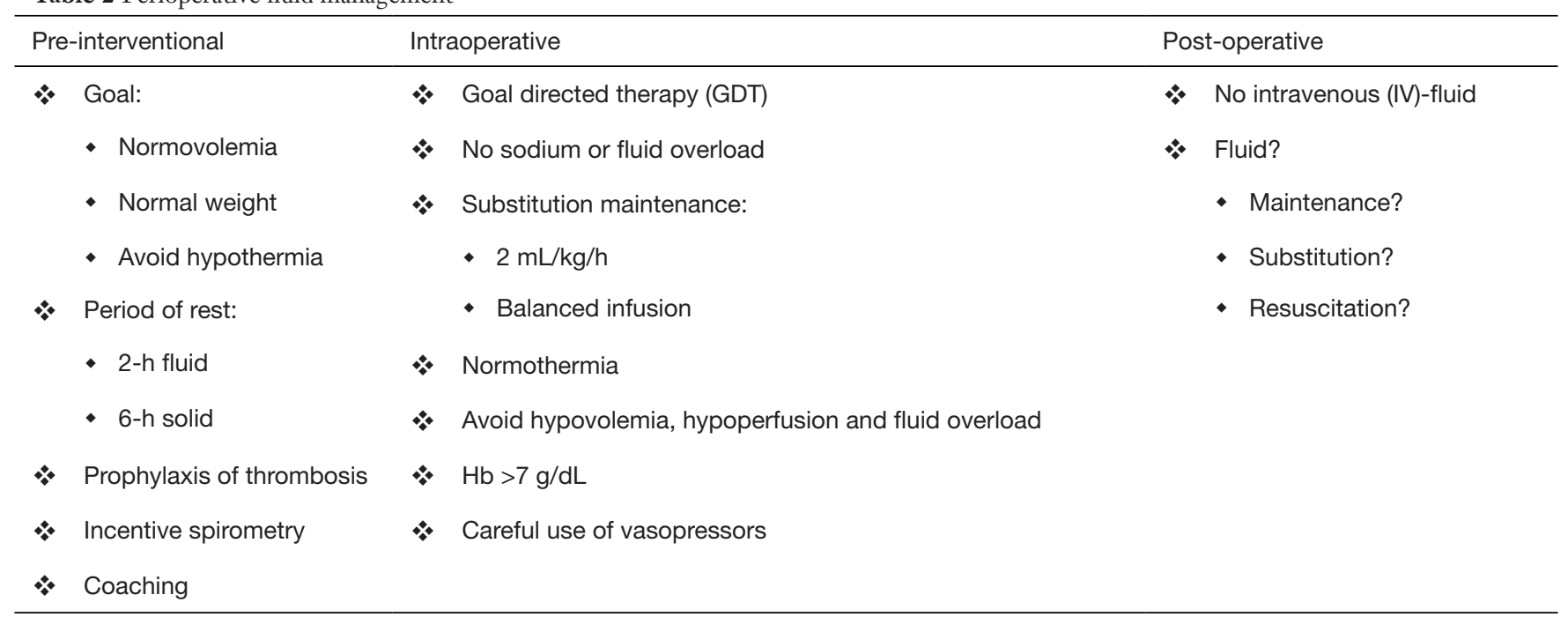

in the outcome of anaemic patients. It should start preoperatively with the substitution of ferritin, vitamin B12 or erythropoietin in patients with low haemoglobin levels $<120 \mathrm{~g} / \mathrm{L}$ in women and $<130 \mathrm{~g} / \mathrm{L}$ in men to avoid the intraoperative use of blood products (28). A blood coagulation management with bedside measurements should take place intraoperative and the administration of any blood products should be avoided (28).

\section{Anaesthesia management for bronchoscopy, Chartis measurement and BLVR}

The procedure of this combined intervention usually requires general anaesthesia with a possible mélange of different techniques in airway management and ventilation. Rigid bronchoscopy, endotracheal intubation, supraglottic airway (SGA) device, and jet ventilation catheters have been used for BLVR. In contrast to standard bronchoscopy, in which the bronchoscope remains in the airway during retrieval of the biopsy specimen, Chartis measurement and valve implementation requires multiple en bloc retrievals of the bronchoscope, the catheter and/or the device. This requires multiple re-navigations through the nose or mouth, may cause epistaxis, and recommends the use of airway protection.

The Chartis measurement, which is an endobronchial pulmonary assessment system, is the preferred and recommended method to measure collateral ventilation in spontaneous breathing or controlled mechanical ventilation with long expiration time and low frequency (29).

Since SGA allows spontaneous breathing or controlled mechanical ventilation and SGA-related complications like mucosal trauma, hematoma, and arytenoid dislocation are rare compared to endotracheal intubation or rigid bronchoscopy (where additional equipment like jet ventilators are needed), SGA devices have numerous well-known advantages (30). The use of the i-gel SGA (Intersurgical Inc., Wokingham, UK) shows fewer complications (i.e., blood staining, sore throat, and dysphagia) compared to other SGA and commonly is used for therapeutic fiberoptic bronchoscopy (31-33). The airway channel cross-section of a size-5 i-gel SGA is elliptical and shows a major axis of $13.4 \mathrm{~mm}$ and a minor axis of $12.9 \mathrm{~mm}$ (33). This results in a cross-section area of $137 \mathrm{~mm}^{2}$ compared to $57 \mathrm{~mm}^{2}$ in a 8.5 -I.D. orotracheal tube. The design of the $\mathrm{i}$-gel ${ }^{\circledast}$, a second-generation SGA with a wide airway channel, facilitates easy passage of the fiberoptic bronchoscope, providing a precise glottic view, easy Chartis measurement, and valve application (34).

To improve bronchoscopist's conditions, general anaesthesia (using remifentanil, propofol and mivacurium) with an i-gel SGA device should be used for BLVR. Jet ventilation through rigid bronchoscopy or with a jet catheter alone may be an alternative concept (respiratory frequency $15-100$ breaths/min, inspiratory pressure 150 $250 \mathrm{kPa})(35)$. 


\section{Postanaesthesia management}

Postinterventional monitoring should detect adverse events like cardiovascular complications (i.e., arrhythmia), pulmonary events (i.e., exacerbation, infection, respiratory failure, and pneumothorax) or valve-related events (i.e., expectoration, migration, aspiration). A 24-h hospitalization on an intermediate care unit may be suggested.

\section{Conclusions}

Anaesthesia plays a key role in the perioperative treatment of patients undergoing LVR. Due to lack of evidence common strategies of anaesthesia in thoracic surgery combined with new procedures can successfully be applied. Deep general anaesthesia and one-lung ventilation through a double-lumen tube are the gold standard in the LVRS, SGA device in the BLVR. New methods like NIVATS are gaining more importance but needs evidence (36).

In the perioperative treatment, the anaesthesiologist can contribute to the result through accurate patient preparation and effective multimodal pain therapy.

\section{Acknowledgements}

None.

\section{Footnote}

Conflicts of Interest: The authors have no conflicts of interest to declare.

\section{References}

1. Benzo R. Lung volume reduction surgery: nonpharmacological approach. Curr Opin Anaesthesiol 2011;24:44-8.

2. Mak PH, Campbell RC, Irwin MG, et al. The ASA Physical Status Classification: inter-observer consistency. American Society of Anesthesiologists. Anaesth Intensive Care 2002;30:633-40.

3. Sharafkhaneh A, Falk JA, Minai OA, et al. Overview of the perioperative management of lung volume reduction surgery patients. Proc Am Thorac Soc 2008;5:438-41.

4. Schulze M, Grande B, Kolbe M, et al. SafAIRway: an airway training for pulmonologists performing a flexible bronchoscopy with nonanesthesiologist administered propofol sedation: A prospective evaluation. Medicine
(Baltimore) 2016;95:e3849.

5. Fleisher LA, Fleischmann KE, Auerbach AD, et al. 2014 ACC/AHA guideline on perioperative cardiovascular evaluation and management of patients undergoing noncardiac surgery: executive summary: a report of the American College of Cardiology/American Heart Association Task Force on practice guidelines. Developed in collaboration with the American College of Surgeons, American Society of Anesthesiologists, American Society of Echocardiography, American Society of Nuclear Cardiology, Heart Rhythm Society, Society for Cardiovascular Angiography and Interventions, Society of Cardiovascular Anesthesiologists, and Society of Vascular Medicine Endorsed by the Society of Hospital Medicine. J Nucl Cardiol 2015;22:162-215.

6. Caviezel C, Aruldas C, Franzen D, et al. Lung volume reduction surgery in selected emphysema patients with pulmonary hypertension. Brompton Session of the 25th European Conference on General Thoracic Surgery in Innsbruck, Austria. 2017.

7. Grønkjær M, Eliasen M, Skov-Ettrup LS, et al. Preoperative smoking status and postoperative complications: a systematic review and meta-analysis. Ann Surg 2014;259:52-71.

8. Haney M, Löfvenberg R, Svensson O. Optimize perioperative health and begin with insistence on preoperative smoking cessation. Acta Anaesthesiol Scand 2014;58:133-4.

9. Musallam KM, Rosendaal FR, Zaatari G, et al. Smoking and the risk of mortality and vascular and respiratory events in patients undergoing major surgery. JAMA Surg 2013;148:755-62.

10. Agostini P, Naidu B, Cieslik H, et al. Effectiveness of incentive spirometry in patients following thoracotomy and lung resection including those at high risk for developing pulmonary complications. Thorax 2013;68:580-5.

11. Zalunardo MP, Ivleva-Sauerborn A, Seifert B, et al. Quality of premedication and patient satisfaction after premedication with midazolam, clonidine or placebo : Randomized double-blind study with age-adjusted dosage. Anaesthesist 2010;59:410-8.

12. Freise H, Van Aken HK. Risks and benefits of thoracic epidural anaesthesia. Br J Anaesth 2011;107:859-68.

13. Popping DM, Elia N, Marret E, et al. Protective effects of epidural analgesia on pulmonary complications after abdominal and thoracic surgery: a meta-analysis. Arch Surg 2008;143:990-9; discussion 1000.

14. Defosse J, Schieren M, Bohmer A, et al. A Germany-wide 
survey on anaesthesia in thoracic surgery. Anaesthesist 2016;65:449-57.

15. Zhao ZR, Lau RW, Ng CS. Non-intubated video-assisted thoracic surgery: the final frontier? Eur J Cardiothorac Surg 2016;50:925-6.

16. Gonzalez-Rivas D, Aymerich H, Bonome C, et al. From Open Operations to Nonintubated Uniportal VideoAssisted Thoracoscopic Lobectomy: Minimizing the Trauma to the Patient. Ann Thorac Surg 2015;100:2003-5.

17. Apfel CC, Heidrich FM, Jukar-Rao S, et al. Evidencebased analysis of risk factors for postoperative nausea and vomiting. Br J Anaesth 2012;109:742-53.

18. Feltracco P, Tonetti T, Barbieri S, et al. Cisatracurium- and rocuronium-associated residual neuromuscular dysfunction under intraoperative neuromuscular monitoring and postoperative neostigmine reversal: a single-blind randomized trial. J Clin Anesth 2016;35:198-204.

19. Beck-Schimmer B, Baumann L, Restin T, et al. Sevoflurane attenuates systemic inflammation compared with propofol, but does not modulate neuro-inflammation: A laboratory rat study. Eur J Anaesthesiol 2017;34:764-75.

20. Beck-Schimmer B, Bonvini JM, Braun J, et al. Which Anesthesia Regimen Is Best to Reduce Morbidity and Mortality in Lung Surgery?: A Multicenter Randomized Controlled Trial. Anesthesiology 2016;125:313-21.

21. Muller-Edenborn B, Frick R, Piegeler T, et al. Volatile anaesthetics reduce neutrophil inflammatory response by interfering with $\mathrm{CXC}$ receptor-2 signalling. $\mathrm{Br} \mathrm{J}$ Anaesth 2015;114:143-9.

22. Colquhoun DA, Naik BI, Durieux ME, et al. Management of 1-Lung Ventilation-Variation and Trends in Clinical Practice: A Report From the Multicenter Perioperative Outcomes Group. Anesth Analg 2018;126:495-502.

23. Huang H, Ma H, Chen S. Enhanced recovery after surgery using uniportal video-assisted thoracic surgery for lung cancer: A preliminary study. Thorac Cancer 2018;9:83-7.

24. Feldheiser A, Aziz O, Baldini G, et al. Enhanced Recovery After Surgery (ERAS) for gastrointestinal surgery, part 2: consensus statement for anaesthesia practice. Acta Anaesthesiol Scand 2016;60:289-334.

25. Li S, Zhou K, Che G, et al. Enhanced recovery programs in lung cancer surgery: systematic review and metaanalysis of randomized controlled trials. Cancer Manag Res 2017;9:657-70.

26. Papadakos PJ, Lachmann B. The open lung concept of mechanical ventilation: the role of recruitment and stabilization. Crit Care Clin 2007;23:241-50, ix-x.

27. Ahn HJ, Kim JA, Lee AR, et al. The Risk of Acute Kidney Injury from Fluid Restriction and Hydroxyethyl Starch in Thoracic Surgery. Anesth Analg 2016;122:186-93.

28. Spahn DR, Goodnough LT. Alternatives to blood transfusion. Lancet 2013;381:1855-65.

29. Klooster K, ten Hacken NH, Hartman JE, et al. Endobronchial Valves for Emphysema without Interlobar Collateral Ventilation. N Engl J Med 2015;373:2325-35.

30. Yu SH, Beirne OR. Laryngeal mask airways have a lower risk of airway complications compared with endotracheal intubation: a systematic review. J Oral Maxillofac Surg 2010;68:2359-76.

31. Park SK, Choi GJ, Choi YS, et al. Comparison of the i-gel and the laryngeal mask airway proseal during general anesthesia: a systematic review and meta-analysis. PLoS One 2015;10:e0119469.

32. Arevalo-Ludeña J, Arcas-Bellas JJ, Alvarez-Rementería R, et al. A comparison of the I-Gel supraglottic device with endotracheal intubation for bronchoscopic lung volume reduction coil treatment. J Clin Anesth 2016;31:137-41.

33. Schmutz A, Dürk T, Idzko M, et al. Feasibility of a Supraglottic Airway Device for Transbronchial Lung Cryobiopsy-A Retrospective Analysis. J Cardiothorac Vasc Anesth 2017;31:1343-7.

34. Theiler L, Gutzmann M, Kleine-Brueggeney M, et al. i-gelTM supraglottic airway in clinical practice: a prospective observational multicentre study. Br J Anaesth 2012;109:990-5.

35. Hubner RH, Herzog D. COPD treatment: about collateral channels and collapsing airways. Eur Respir J 2016;47:1606-10.

36. Hung WT, Liao HC, Cheng YJ, et al. Nonintubated Thoracoscopic Pneumonectomy for Bullous Emphysema. Ann Thorac Surg 2016;102:e353-5.
Cite this article as: Grande B, Loop T. Anaesthesia management for bronchoscopic and surgical lung volume reduction. J Thorac Dis 2018;10(Suppl 23):S2738-S2743. doi: 10.21037/jtd.2018.02.46 